\title{
Squeezing Phase Diffusion
}

\author{
Simone Cialdi ${ }^{1,2}$ Edoardo Suerra $\odot^{1,2}$ Stefano Olivares $\odot,{ }^{1,2}$ Stefano Capra, ${ }^{1,2}$ and Matteo G. A. Paris $\oplus^{1,2}$ \\ ${ }^{1}$ Dipartimento di Fisica "Aldo Pontremoli," Università degli Studi di Milano, I-20133 Milano, Italia \\ ${ }^{2}$ Istituto Nazionale di Fisica Nucleare, Sezione di Milano, I-20133 Milano, Italia
}

(Received 14 July 2019; accepted 6 April 2020; published 23 April 2020)

\begin{abstract}
We address the use of optical parametric oscillator (OPO) to counteract phase diffusion, and demonstrate phase-noise reduction for coherent signals traveling through a suitably tuned OPO. In particular, we theoretically and experimentally show that there is a threshold value on the phase noise, above which OPO can be exploited to "squeeze" phase noise. The threshold depends on the energy of the input coherent state, and on the relevant parameters of the OPO, i.e., gain and input-output and crystal loss rates.
\end{abstract}

DOI: 10.1103/PhysRevLett.124.163601

Introduction.-The phase of a harmonic oscillator is not an observable in a strict sense, and this fundamental feature makes it challenging to analyze phase manipulation, amplification, and detection at the quantum level. At the same time, the phase of an optical signal represents a basic building block for several applications in quantum optics and metrology [1-10], and protocols based on phase-shiftkeyed coherent signals are useful in those scenarios where single-photon and entangled states may not be the optimal choice, as it happens in free-space communication. In these protocols, the major obstacle to fully exploit the advantages of quantum measurements, e.g., beating the shot-noise limit, is phase noise due to phase diffusion [11-17].

A question thus arises on whether, and how, it may be possible to contrast and counteract the effects of phase diffusion, and to decrease its detrimental effect on the coherence of the signal. In this framework, a natural choice to hamper phase diffusion is to use phase-sensitive amplification, as that provided by optical parametric oscillators (OPOs) in the degenerate regime. Despite the simplicity of the underlying idea the use of OPO to squeeze phase diffusion did not receive much attention in the past. The reason is twofold: on the theoretical side, the existence of quantum limits to amplification suggests that no full compensation of noise is possible [18-22]. In addition, OPO is amplifying field quadratures and not the phase itself, which is not an observable in a strict sense. On the experimental side, seeding a quantum amplifier with a phase-diffused state in order to compensate the noise is not a trivial task, though there have been efforts in this direction $[23,24]$. Likewise, achieving the necessary level of pump phase stabilization is a fundamental issue and a challenging task in itself.

Here, thanks to an active stabilization scheme of our OPO and to a novel technique for pump stabilization, we have been able to experimentally address the use of optical parametric oscillator (OPO) to counteract phase noise, and to demonstrate reduction of phase diffusion for coherent signals. In particular, we theoretically and experimentally show that there is a threshold value on the phase noise, above which OPOs may be exploited to effectively squeeze phase noise. As we will see, the noise threshold depends on the amplitude of the input signal, and on the relevant parameters of the OPO, i.e., gain and input or output and crystal loss rates.

$O P O$ and phase diffusion. - Let us consider a coherent state $\left|\beta e^{i \varphi}\right\rangle$, with $\beta \in \mathbb{R}_{+}$undergoing phase diffusion. The evolved state may be written as $[15,25]$

$$
\varrho=\int d \phi g_{\sigma}(\phi)\left|\beta e^{i \phi}\right\rangle\left\langle\beta e^{i \phi}\right|,
$$

where $g_{\sigma}(\phi)$ is a Gaussian distribution $g_{\sigma}(\phi)=\left(2 \pi \sigma^{2}\right)^{-1 / 2} \times$ $\exp \left\{-\frac{1}{2} \phi^{2} / \sigma^{2}\right\}$. We refer to $\sigma$ as to the amplitude of the phase diffusion (see Fig. 1).

An estimate of the phase $\varphi$ may be obtained from the expectations of the two orthogonal quadratures $x=a+a^{\dagger}$ and $y=i\left(a^{\dagger}-a\right)$, where $a$ and $a^{\dagger}$ are the creation and annihilation operators, $\left[a, a^{\dagger}\right]=1$. By exploiting the

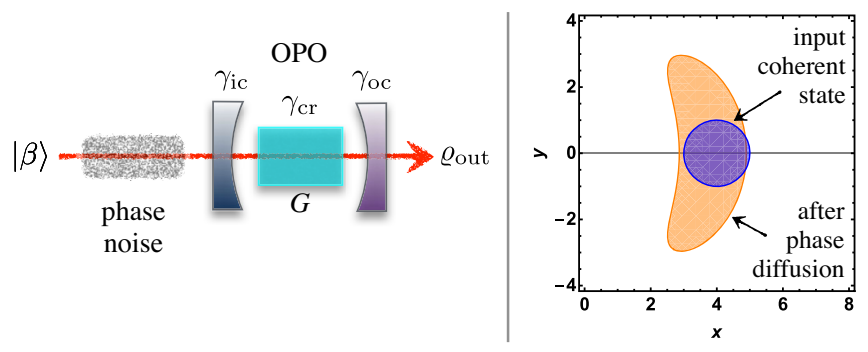

FIG. 1. (Left panel): After the phase diffusion the noisy signal enters an OPO characterized by the gain $G$ and by the input $\gamma_{\text {ic }}$, output $\gamma_{\mathrm{oc}}$, and crystal $\gamma_{\mathrm{cr}}$ loss rates. (Right panel): phase-space representation of a coherent state $|\beta\rangle$ (blue) and its phase-diffused counterpart (orange). We set $\beta=2$ and $\sigma=\pi / 4$. It is clear the effect of phase noise on the uncertainty of the $x$ and $y$ quadratures. 
stability of our setup, we estimate phase by two measurements performed on two successive preparations, of the input state [26], i.e., $\hat{\varphi}=\arctan [\langle y\rangle /\langle x\rangle]$, thus avoiding any extra noise due to joint measurement of quadratures [27]. For the range of parameters we consider in our experiment (i.e., $\hat{\varphi}=0$ and $\delta \phi \lesssim 0.5$ ), the uncertainty $\delta \phi^{2} \equiv \operatorname{var}[\hat{\varphi}]$ in the estimate $\hat{\varphi}$ is thus given by $\delta \phi^{2}=\left\{\langle y\rangle^{2} \operatorname{var}[x]+\right.$ $\left.\langle x\rangle^{2} \operatorname{var}[y]\right\} / \Xi^{4}$, where $\Xi^{2}=\langle y\rangle^{2}+\langle x\rangle^{2}$. For a coherent signal we have $\operatorname{var}[x]=\operatorname{var}[y]=1$ and thus $\delta \phi_{0}^{2}=\left(4 \beta^{2}\right)^{-1}$, i.e., shot-noise scaling. Let us now consider a coherent signal with amplitude $\beta \in \mathbb{R}_{+}$undergoing phase diffusion (see Fig. 1). In this case we have $\langle x\rangle=2 \beta e^{(-1 / 2) \sigma^{2}}$, $\langle y\rangle=0, \quad \operatorname{var}[x]=1+2 \beta^{2}\left(1-e^{-\sigma^{2}}\right)^{2}$, and $\operatorname{var}[y]=1+$ $2 \beta^{2}\left(1-e^{-2 \sigma^{2}}\right)$, with $\operatorname{var}[x] \leq \operatorname{var}[y]$. It is straightforward to show that

$$
\delta \phi_{\sigma}^{2}=\frac{\cosh \left(\sigma^{2}\right)+\left(1+4 \beta^{2}\right) \sinh \left(\sigma^{2}\right)}{4 \beta^{2}} \geq \delta \phi_{0}^{2} .
$$

Let us now assume that after the phase-diffusion process the degraded state passes through an OPO as sketched in the left panel of Fig. 1. The OPO is characterized by the value of its gain, by the input or output parameters $\eta_{\text {in }}$ and $\eta_{\text {esc }}$, which summarize the effect of the transmissivity of the input and output couplers, and of the internal losses [28]. The gain can be written as $G=(1-d)^{-2}$, where $d=\sqrt{P / P_{\text {th }}}$, where $P$ is the pump power and $P_{\text {th }}$ the OPO power threshold. The input and output parameters depend on the loss rates at the couplers at the input $\left(\gamma_{\text {ic }}\right)$ and the output $\left(\gamma_{\mathrm{oc}}\right)$, and also on the loss rate due to the OPO crystal $\left(\gamma_{\text {cr }}\right)$. Overall, we have

$$
\eta_{\text {in }}=\gamma_{\text {ic }} / \gamma \quad \eta_{\text {esc }}=\gamma_{\mathrm{oc}} / \gamma,
$$

where the global loss rate is given by $\gamma=\gamma_{\mathrm{ic}}+\gamma_{\mathrm{oc}}+2 \gamma_{\mathrm{cr}}$. In the following, we will focus on the effect of the OPO on the first and second moments of the quadratures.

Let us start by considering an input coherent state $\left|\beta e^{i \phi}\right\rangle$ (without phase noise). By lengthy but straightforward calculations we obtain $X \equiv\langle x\rangle=\alpha_{x} \cos \varphi, \quad Y \equiv\langle y\rangle=$ $\alpha_{y} \sin \varphi$ (we choose the OPO pump phase such to amplify the $x$ quadrature), where $\alpha_{x / y}=\sqrt{4 \eta_{\text {in }} \eta_{\text {esc }}} 2 \beta /(1 \mp d)$ and

$\Sigma_{x / y}^{2}\left(\eta_{\mathrm{esc}}, d\right) \equiv \operatorname{var}[x / y]=1 \pm 4 d \eta_{\mathrm{esc}} /(1 \mp d)^{2} \geq 1$.

In Fig. 2 we show the effect of the OPO on a coherent state for a particular choice of the involved parameters. Notice that besides squeezing, the OPO reduces the phase shift of the state.

If phase noise occurs during the propagation of the coherent state before the OPO, the evolved state is given by Eq. (1). The mean values of quadratures after the OPO and their variances are now given by $\langle x\rangle=\alpha_{x} e^{(-1 / 2) \sigma^{2}},\langle y\rangle=0$, and
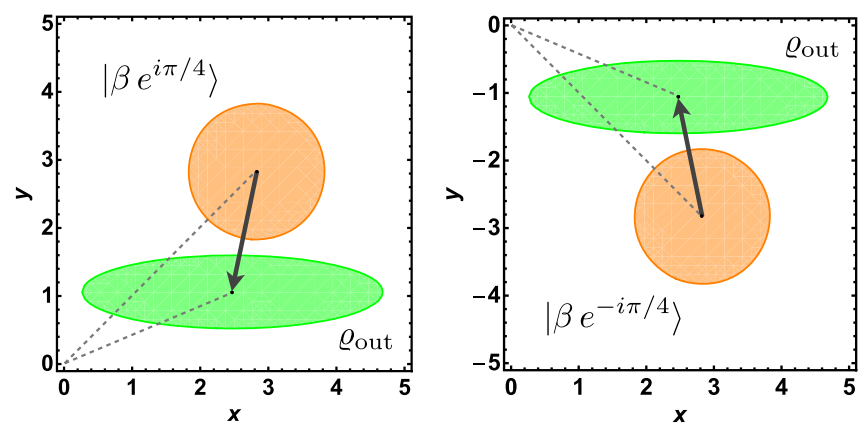

FIG. 2. Phase-space representation of a coherent $\left|\beta e^{i \varphi}\right\rangle$ state before (orange) and after the OPO (green). We set $\beta=2$ and two values of the phase: (a) $\varphi=\pi / 4$ and (b) $\varphi=-\pi / 4$. We used realistic OPO parameters $d=0.40(G=2.78), \eta_{\text {in }}=0.08$, and $\eta_{\mathrm{esc}}=0.87$.

$$
\operatorname{var}[x / y]=\Sigma_{x / y}^{2}+\alpha_{x / y}^{2}\left(1 \pm e^{-2 \sigma^{2}}\right) / 2-g_{x / y},
$$

where $g_{x}=e^{-\sigma^{2}}, g_{y}=0$, and the $\Sigma_{x / y}^{2}$ are given in Eq. (4).

In Fig. 3 we can see how a phase-diffused coherent state is modified by the evolution through the OPO. In order to assess the effects of OPO, and to quantify the possible reduction of phase diffusion, we evaluate the phase variance at the output. Using the above expressions for the moments we have

$$
\delta \phi_{\mathrm{OPO}}^{2}=\Sigma_{y}^{2} / \alpha_{x}^{2} e^{\sigma^{2}}+\alpha_{y}^{2} / \alpha_{x}^{2} \sinh \sigma^{2} .
$$

Upon comparing the variance (6) to that before the OPO, i.e., Eq. (2), we find a threshold value $\sigma_{\text {th }} \equiv \sigma_{\text {th }}\left(\beta, \eta_{\text {in }}\right.$, $\left.\eta_{\mathrm{esc}}, d\right)$ on the phase noise, above which phase diffusion is reduced. More explicitly, we have that $\gamma_{\sigma}=\delta \phi_{\mathrm{OPO}}^{2} / \delta \phi_{\sigma}^{2}<1$ iff $\sigma^{2}>\sigma_{\mathrm{th}}^{2}$, where $\sigma_{\mathrm{th}}^{2}=\frac{1}{2} \log \left\{2 \beta^{2}\left(\alpha_{x}^{2}-\alpha_{y}^{2}\right) /\left[\alpha_{x}^{2}+2 \beta^{2}\left(\alpha_{x}^{2}-\right.\right.\right.$ $\left.\left.\left.\alpha_{y}^{2}-2 \Sigma_{y}^{2}\right)\right]\right\}$. In Fig. 4 we plot the threshold $\sigma_{\text {th }}$ as a function of the OPO gain $G=(1-d)^{-2}$ for different values of the
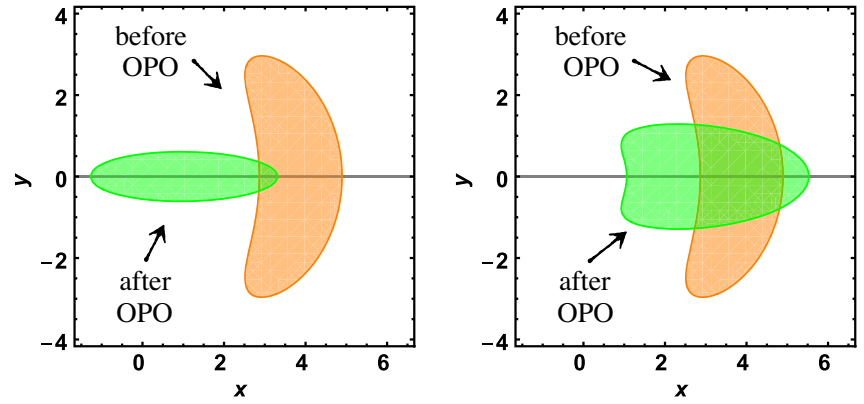

FIG. 3. Phase-space representation of a coherent state after phase diffusion (orange) and after the OPO (green). The initial coherent amplitude is $\beta=2$ and $\varphi=0$. The phase-noise amplitude is $\sigma=\pi / 4$, whereas $d=0.4(G=2.78)$. We used the realistic OPO values (a) $\eta_{\text {in }}=0.01, \quad \eta_{\text {esc }}=0.93$ and (b) $\eta_{\text {in }}=0.08, \eta_{\text {esc }}=0.87$. Fluctuations of the $y$ quadrature are reduced after the evolution through the OPO. 

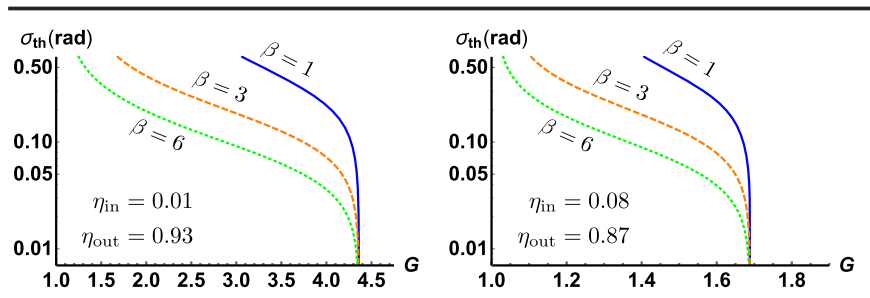

FIG. 4. Threshold value $\sigma_{\text {th }}$ on the phase noise as a function of the OPO gain $G=(1-d)^{-2}$ for different values of the input coherent state $\beta$ : if $\sigma>\sigma_{\text {th }}$ the OPO reduces the phase noise. The input and output parameters are the following: $\eta_{\text {in }}=0.01$ and $\eta_{\text {esc }}=0.93$ (left panel) and $\eta_{\text {in }}=0.08$ and $\eta_{\text {esc }}=0.87$ (right panel). We cut the vertical axis in order to have $\sigma_{\text {th }} \lesssim 0.5$, where our analysis based on the error propagation holds.

involved parameters. We can see that there always exists a maximum value of $G$, above which the OPO always reduces phase noise. For large gain $G$, or large amplitude $\beta$ (keeping in mind that $\beta \in \mathbb{R}_{+}$), we have $\gamma_{\sigma} \approx G^{-1}$.

Experimental results.-Theoretical predictions have been tested using the experimental scheme depicted in Fig. 5. The setup allows us to generate and manipulate displaced-squeezed states. In particular, we have control not only of the amplitude and phase of the states, but also of the gain $G$ and the pump phase. The basic elements of the setup are described in detail in [29]. Overall, it consists of three blocks: a Laser source (LASER), a state generation and manipulation stage (SG), and a homodyne detector (HD). A homemade $1064 \mathrm{~nm}$ wavelength Nd:YAG laser internally frequency doubled at $532 \mathrm{~nm}$ serves both as the input seed and the pump beam for the optical parametric oscillator (OPO). In particular, from the infrared output of the laser we generate two different beams by a polarizing beam splitter: one is used as the local oscillator (LO) for the homodyne detection, while the other is sent to the SG.

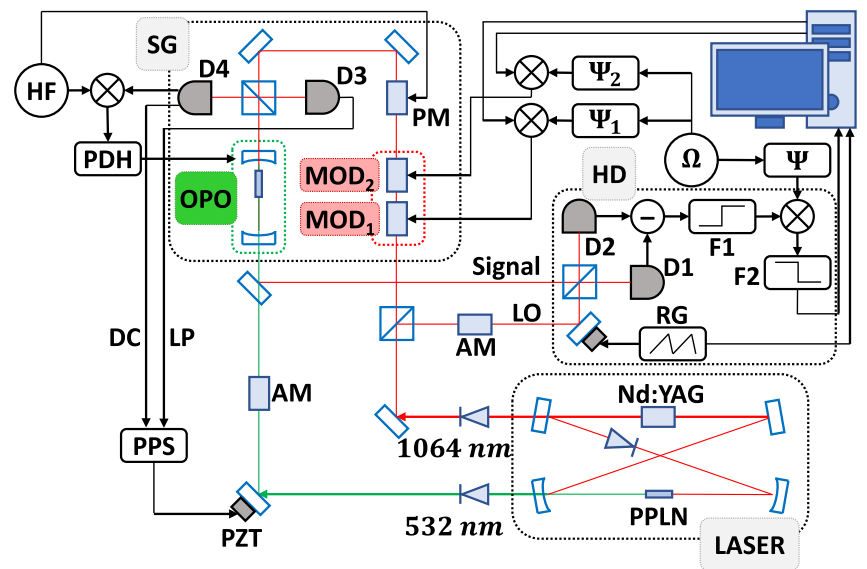

FIG. 5. Schematic diagram of the experimental setup. The main source is the Nd:YAG laser internally frequency doubled, while $\mathrm{OPO}$ is in the state generation stage (SG). States are revealed by a homodyne detector (HD). Generation and detection are fully controlled by a computer. See the text and Ref. [29] for further details.
Our OPO consists of a linear cavity with a free spectral range of $3.270 \mathrm{GHz}$. A $10 \mathrm{~mm}$-long $\mathrm{MgO}: \mathrm{LiNbO} 3$ crystal with antireflection coating is inserted inside the cavity. The losses related to the crystal are $\Delta=2.42 \times 10^{-3}$. We used two different configurations $A$ and $B$ for the OPO. In configuration $A$ the input mirror has a reflectivity $R_{\mathrm{ic}, A}=$ 0.999 with a radius of curvature of $10 \mathrm{~mm}$, while the output mirror has a reflectivity $R_{\mathrm{oc}}=0.917$ with a radius of curvature of $25 \mathrm{~mm}$, leading to $\eta_{\mathrm{in}, A}=0.008$ and $\eta_{\text {esc }, A}=0.937$, with a measured cavity total transmissivity $T_{A}=0.029$. In configuration $B$ the input mirror has a reflectivity $R_{\mathrm{ic}, B}=0.9925$, and the output mirror is the same as $A$, both with the same radii of curvature of $A$, leading to $\eta_{\mathrm{ic}, B}=0.079$ and $\eta_{\mathrm{esc}, B}=0.871$, with a measured cavity total transmissivity $T_{B}=0.26$ (the role of the transmissivities will be clarified later on). The cavity is actively stabilized using the Pound-Drever-Hall (PDH) technique [30] by means of a phase modulator (PM) placed along the SG beam, which generates two $116 \mathrm{MHz}$ sidebands around the laser frequency. Coherent states are generated exploiting the combined effect of two optical modulators (MOD1 and MOD2) placed before the OPO. A proper choice of their modulations allows us to generate an arbitrary coherent state on the sidebands at $3 \mathrm{MHz}$ for seeding the OPO $[29,30]$. Amplitude and phase values are set on demand by a computer.

In order to effectively amplify a certain quadrature with the OPO, the pump phase $\Theta$ must be stable over the whole time of the measurement. To this aim, a novel technique for pump phase stabilization (PPS) has been developed [31], which will be briefly described in the following. In our stabilization technique, the field $E_{r}^{(\text {tot })}$ reflected by the OPO can be exploited as an error signal for the stabilization of $\Theta$. We note that the field $E_{r}^{(\text {tot })}$ is the sum of the field $E_{r}$, directly reflected by the input mirror, and the field $E_{t}$ transmitted by the cavity through the input mirror. The laser and the field generated by down conversion by the pump interact inside the cavity and their interaction leads to constructive or destructive interference depending on the pump phase $\Theta$. Since $E_{r}$ and $E_{t}$ are $\pi$ shifted, in the first case $E_{t}$ increases and $E_{r}^{(\text {tot })}$ decreases, while in the second case $E_{t}$ decreases and $E_{r}^{(\text {tot })}$ increases. This can be summarized as $E_{r}^{(\mathrm{tot})}=E_{r}^{(\mathrm{tot})}(\Theta)$, thus in order to access the information about $\Theta$ we measure the corresponding power $P_{r}^{(\text {tot })}$ with the dc output of the detector $D_{4}$ (see Fig. 5). In order to improve the performances of the stabilization system, the amplitude noise of the laser is removed from the PPS error signal by means of detector $D_{3}$ (i.e., a laser power monitor) and of dedicated electronics. Finally, the obtained error signal is properly manipulated with a proportionalintegral-derivative controller, and applied to a piezoelectric actuator which may tune $\Theta$. The total bandwidth of the PPS in our setup is about $1 \mathrm{kHz}$ and, in turn, we may 
compensate pump phase fluctuations of at most that frequency. Notice that the PPS has been performed using the career frequency of the seeding beam. This enables one to prepare a coherent state with arbitrary amplitude and phase on the sidebands at $3 \mathrm{MHz}$, independently of the PPS scheme.

The effect of the OPO on a phase-diffused coherent state has been tested directly by generating a rapid sequence of three coherent states: one with no phase shift, and two with a $\pm 40^{\circ}$ phase shift. Since after the OPO we have $\langle y\rangle /\langle x\rangle=(1-d) /(1+d) \tan \varphi$, the mean phase $\theta_{d}$ after the OPO may be found from the value $\theta$ before the OPO by the relation

$$
\tan \theta_{d}=\tan \theta(1-d) /(1+d) .
$$

We performed homodyne detection on every state, with and without the OPO. Results are shown in the upper panels of Fig. 6. In order to highlight the effect of the OPO on the phase shift, we used two different amplitudes for the cases with and without the OPO since. As it follows from Eq. (7) this is irrelevant, since the reduction of the phase shifts does not depend on the $\beta$ s. In particular, in configuration $A$ we had $\beta=4.5$ without $\mathrm{OPO}$ and $\beta_{\mathrm{OPO}}=2.0$ with $\mathrm{OPO}$; the gain was $G=3.1$, leading to $d=0.43$. Results show that the phase shift is reduced from $\theta=40^{\circ}$ to a measured $\theta_{d, \mathrm{ex}}=20^{\circ}$, while the theoretical value is $\theta_{d, \text { th }}=18.4^{\circ}$.

In our experiments, we generated the phase-diffused coherent states by modulating their phases with a suitable Gaussian distribution [32] and evaluated $\operatorname{var}[\hat{\varphi}]$ of Eq. (6) with and without OPO for different values of the phase diffusion $\sigma$ in both configurations $A$ and $B$. In order to preserve stability and reproducibility, in the measurements without OPO we did not physically remove it from the setup, but rather we increased the amplitude of the coherent state by a factor of $1 / \sqrt{T}$ with respect to the case of squeezed coherent, in order to compensate the effect of the cavity transmissivity $T$. This is why we carefully measured transmissivities. Of course, pump was turned off during these measurements. In order to obtain $\operatorname{var}[\hat{\varphi}]$, we measured $\langle x\rangle,\langle y\rangle, \operatorname{var}[x]$, and $\operatorname{var}[y]$ and calculated $\delta \phi^{2}$. We made measurements for different values of the phase diffusion $\sigma$. The theoretical expectation has been obtained directly using Eq. (6), where we considered the experimental values for $\Sigma_{y}, \beta$, and $G$, from which we calculated $\alpha_{x}$ and $\alpha_{y}$ and then threshold $\sigma_{\mathrm{th}}$. Both the experimental points and the theoretical previsions are shown in the lower panels of Fig. 6 for the two configurations. In particular, in configuration $A$ we have $\beta_{A}=5.70$ and $G_{A}=2.75$, with a theoretical threshold $\sigma_{\text {th }}=14.8^{\circ}$ perfectly compatible to experimental points. In configuration $B$ we have $\beta_{B}=2.05, G_{B}=3.12$ and there is no threshold, so the use of the OPO is convenient for every phase-diffusion amplitude.
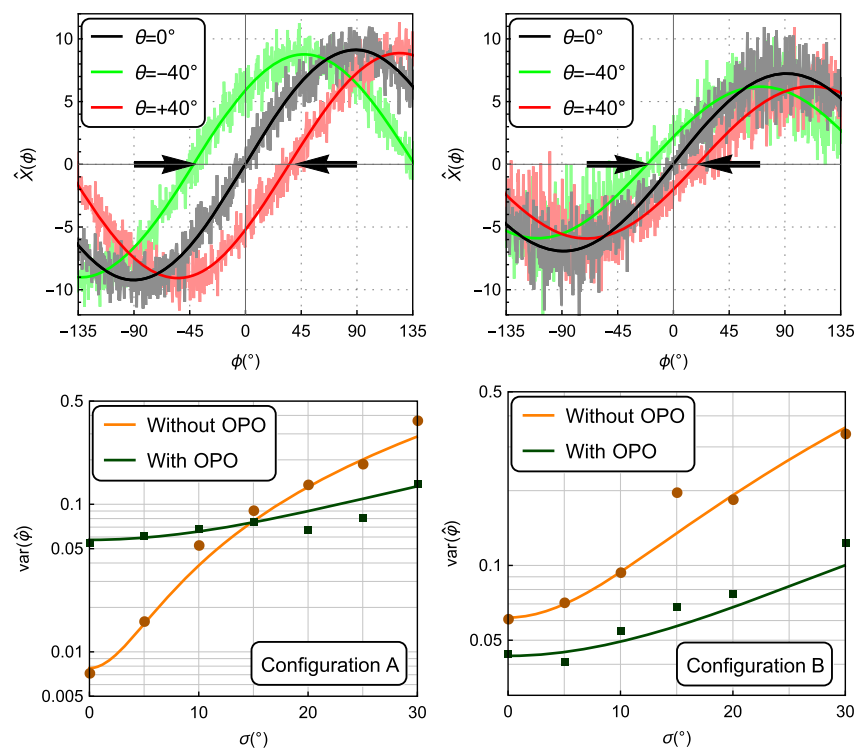

FIG. 6. (Upper panels): Homodyne traces for (left) three coherent states with $\theta=-40^{\circ}, 0^{\circ}$, and $+40^{\circ}$ and (right) the corresponding states after the OPO. The reduction of the phase shift is marked by the arrows. The amplitudes are $\beta=4.5$ and $\beta_{\mathrm{OPO}}=2.0$ with $G=3.1$. (Lower panels): Phase variance as a function of the phase diffusion for the configurations $A$ (left) and $B$ (right). Theoretical curves (lines) fit well the experimental points. Notice the presence of the threshold at $14.8^{\circ}$ in configuration $A$.

Discussion.-In order to reduce phase noise we are operating our OPO in the deamplification regime and this means that besides noise also the mean value of the phase is reduced, see, e.g., Eq. (7). As a consequence, the signal-tonoise ratio is not necessarily increased at the output of the $\mathrm{OPO}$, and a question arises on whether our device may be exploited in practical scenarios. As a matter of fact, squeezing and phase-noise reduction are not expected to be useful to build receivers, i.e., in an interferometer or in the detection stage of a communication scenario. Rather, it finds applications in the preparation stage, to contrast and counteract the effect of phase diffusion. In particular, if we consider a phase estimation (or communication) protocol in which a phase shift is applied to a phase-diffused seed coherent state, i.e., phase diffusion affects the generation stage of the protocol (before the phase encoding), we expect that squeezing may counteract the detrimental effects of phase noise. In turn, it improves the overall performance of the seed state in the phase estimation scheme and preliminary results confirm our prevision [33]. The full analysis requires analysis of the problem by quantum estimation theory and it will be thoroughly addressed in a forthcoming work [33]. On the other hand, it should be noticed that if the squeezing is used in the detection stage, namely, it is applied after the encoding stage (and, thus, also after phase diffusion), it may be no longer useful, since the resulting reduction of the phase 
variance, due to squeezing, is vanished by the modification occurring also to the phase mean value.

Conclusions. - In conclusion, using our innovative OPO scheme we have been able to exploit phase-sensitive amplification to counteract phase noise. In particular, our results demonstrate the reduction of phase diffusion for coherent signals traveling through a suitably tuned OPO. More generally, we have shown that there is a threshold value on the phase noise, above which OPO can be exploited to squeeze phase noise. The threshold depends on the energy of the input coherent state, and on the relevant parameters of the OPO. Our results may be exploited for state preparation in quantum metrological schemes and to enhance quantum phase communication channels, e.g., using adaptive schemes [10] exploiting the reduction of phase noise, as well as the reduction of phase signal.

Useful discussions with M. G. Genoni and G. Carrara are kindly acknowledged. This work has been supported by UniMI, Project No. PSR2017-DIP-008, and by MAECI, Project No. PGR06314-ENYGMA.

[1] C. M. Caves, Quantum-mechanical noise in an interferometer, Phys. Rev. D 23, 1693 (1981).

[2] M. Holland and K. Burnett, Interferometric Detection of Optical Phase Shifts at the Heisenberg Limit, Phys. Rev. Lett. 71, 1355 (1993).

[3] V. Giovannetti, S. Lloyd, and L. Maccone, Quantum Metrology, Phys. Rev. Lett. 96, 010401 (2006).

[4] V. Giovannetti, S. Lloyd, and L. Maccone, Advances in quantum metrology, Nat. Photonics 5, 222 (2011).

[5] R. Demkowicz-Dobrzański, U. Dorner, B. J. Smith, J. S. Lundeen, W. Wasilewski, K. Banaszek, and I. A. Walmsley, Quantum phase estimation with lossy interferometers, Phys. Rev. A 80, 013825 (2009).

[6] B. Escher, R. de Matos Filho, and L. Davidovich, General framework for estimating the ultimate precision limit in noisy quantum-enhanced metrology, Nat. Phys. 7, 406 (2011).

[7] R. Chaves, J. Brask, M. Markiewicz, J. Kołodyński, and A. Acn, Noisy Metrology Beyond the Standard Quantum Limit, Phys. Rev. Lett. 111, 120401 (2013).

[8] A. Smirne, J. Kołodyński, S. F. Huelga, and R. DemkowiczDobrzański, Ultimate Precision Limits for Noisy Frequency Estimation, Phys. Rev. Lett. 116, 120801 (2016).

[9] S. Daryanoosh, S. Slussarenko, D. W. Berry, H. M. Wiseman, and G. J. Pryde, Experimental optical phase measurement approaching the exact Heisenberg limit, Nat. Commun. 9, 4606 (2018).

[10] A. A. Berni, T. Gehring, Bo M. Nielsen, V. Handchen, M. G. A. Paris, and U. L. Andersen, Ab-initio quantum enhanced optical phase estimation using real-time feedback control, Nat. Photonics 9, 577 (2015).

[11] M. Bina, A. Allevi, M. Bondani, and S. Olivares, Phasereference monitoring in coherent-state discrimination assisted by a photon-number resolving detector, Sci. Rep. 6, 26025 (2016).

[12] C. Wittmann, U. L. Andersen, M. Takeoka, D. Sych, and G. Leuchs, Demonstration of Coherent-State Discrimination Using a Displacement-Controlled Photon-NumberResolving Detector, Phys. Rev. Lett. 104, 100505 (2010).

[13] C. R. Müller, M. A. Usuga, C. Wittmann, M. Takeoka, C. Marquardt, U. L. Andersen, and G. Leuchs, Quadrature phase shift keying coherent state discrimination via a hybrid receiver, New J. Phys. 14, 083009 (2012).

[14] S. Izumi, M. Takeoka, K. Ema, and M. Sasaki, Quantum receivers with squeezing and photon-number-resolving detectors for $M$-ary coherent state discrimination, Phys. Rev. A 86, 042328 (2012).

[15] M. G. Genoni, S. Olivares, and M. G. A. Paris, Optical Phase Estimation in the Presence of Phase Diffusion, Phys. Rev. Lett. 106, 153603 (2011).

[16] C. Wittmann, M. Takeoka, K. N. Cassemiro, M. Sasaki, G. Leuchs, and U. L. Andersen, Demonstration of NearOptimal Discrimination of Optical Coherent States, Phys. Rev. Lett. 101, 210501 (2008).

[17] K. Tsujino, D. Fukuda, G. Fujii, S. Inoue, M. Fujiwara, M. Takeoka, and M. Sasaki, Quantum Receiver Beyond the Standard Quantum Limit of Coherent Optical Communication, Phys. Rev. Lett. 106, 250503 (2011).

[18] W. H. Louisell, A. Yariv, and A. E. Siegman, Quantum fluctuations and noise in parametric processes I, Phys. Rev. 124, 1646 (1961); J. P. Gordon, W. H. Louisell, and L. R. Walker, Quantum fluctuations and noise in parametric processes II, Phys. Rev. 129, 481 (1963).

[19] H. Heffner, The fundamental noise limit of linear amplifiers, Proc. IRE 50, 1604 (1962).

[20] H. A. Haus and J. A. Mullen, Quantum noise in linear amplifiers, Phys. Rev. 128, 2407 (1962).

[21] C. M. Caves, Quantum limits on noise in linear amplifiers, Phys. Rev. D 26, 1817 (1982).

[22] C. M. Caves, J. Combes, Z. Jiang, and S. Pandey, Quantum limits on phase-preserving linear amplifiers, Phys. Rev. A 86, 063802 (2012).

[23] R. Filip, P. Marek, and U. L. Andersen, Measurementinduced continuous-variable quantum interactions, Phys. Rev. A 71, 042308 (2005).

[24] J. I. Yoshikawa, T. Hayashi, T. Akiyama, N. Takei, A. Huck, U. L. Andersen, and A. Furusawa, Demonstration of deterministic and high fidelity squeezing of quantum information, Phys. Rev. A 76, 060301(R) (2007).

[25] M. G. Genoni, S. Olivares, D. Brivio, S. Cialdi, D. Cipriani, A. Santamato, S. Vezzoli, and M. G. A. Paris, Optical interferometry in the presence of large phase diffusion, Phys. Rev. A 85, 043817 (2012).

[26] S. Olivares, Quantum optics in the phase space-A tutorial on Gaussian states, Eur. Phys. J. Special Topics 203, 3 (2012).

[27] H. P. Yuen, Generalized quantum measurements and approximate simultaneous measurements of noncommuting observables, Phys. Lett. A 91, 101 (1982).

[28] H.-A. Bachor and T. C. Ralph, A Guide to Experiments in Quantum Optics (Wiley-VCH, Weinheim, 2004).

[29] A. Mandarino, M. Bina, C. Porto, S. Cialdi, S. Olivares, and M. G. A. Paris, Assessing the significance of fidelity as a 
figure of merit in quantum state reconstruction of discrete and continuous variable systems, Phys. Rev. A 93, 062118 (2016).

[30] S. Cialdi, C. Porto, D. Cipriani, S. Olivares, and M. G. A. Paris, Full quantum state reconstruction of symmetric twomode squeezed thermal states via spectral homodyne detection and a state-balancing detector, Phys. Rev. A 93, 043805 (2016).
[31] S. Cialdi, E. Suerra, and S. Olivares, Pump phase stabilisation in an optical parametric oscillator (to be published).

[32] S. Olivares, S. Cialdi, F. Castelli, and M. G. A. Paris, Homodyne detection as a near-optimum receiver for phase-shift keyed binary communication in the presence of phase diffusion, Phys. Rev. A 87, 050303(R) (2013).

[33] G. Carrara, M. G. Genoni, M. G. A. Paris, S. Cialdi, and S. Olivares (to be published). 